\title{
The Relationship between Weight Status and Depressive Symptoms in a Population Sample with Obesity: The Mediating Role of Appearance Evaluation
}

\author{
Natascha-Alexandra Weinberger ${ }^{a, d} \quad$ Anette Kersting $^{b} \quad$ Steffi G. Riedel- \\ Heller $^{c}$ Claudia Luck-Sikorski ${ }^{a}{ }^{d}$ \\ aIntegrated Research and Treatment Center (IFB) AdiposityDiseases, University Hospital

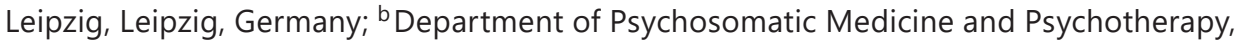 \\ University Hospital Leipzig, Leipzig, Germany; ' Institute of Social Medicine, Occupational \\ Health and Public Health (ISAP), University Hospital Leipzig, Leipzig, Germany; ${ }^{d}$ University \\ of Applied Health Sciences SRH Gera, Gera, Germany
}

\section{Keywords}

Appearance evaluation · Body image $\cdot$ Obesity $\cdot$ Depressive symptoms · Adults

\begin{abstract}
Objectives: Identifying the underlying pathways between obesity and depression and which individuals with obesity are at risk for developing depressive symptoms is important for improving prevention and treatment efforts. The current study investigated appearance evaluation as a potential mediator of the relationship between obesity and depression. Methods: A total of 1,000 participants with a self-reported BMI greater than $30 \mathrm{~kg} / \mathrm{m}^{2}$ were interviewed about their experiences with an elevated body weight and completed measures on appearance evaluation and depression. Results: A negative relationship between satisfaction with appearance and $\mathrm{BMI}$ and a negative association between satisfaction with appearance and depression was found in men and women. Men reported less depressive symptoms and higher satisfaction with appearance compared to women. While no mediation effect could be determined in women, satisfaction with appearance mediated the obesity-depression relationship in men. Conclusions: Efforts to reduce adverse effects of either obesity or depression need to take the reciprocal relationship between the two conditions into account. The current findings underscore the importance of appearance evaluation for treatment efforts in individuals with obesity. Interventions addressing body image might improve both, psychological distress and weight management efforts in general.


Weinberger et al.: The Relationship between Weight Status and Depressive Symptoms in a Population Sample with Obesity: The Mediating Role of Appearance Evaluation

\section{Introduction}

Obesity is widely considered a major public health issue, and its adverse physical consequences have been well documented [1]. More recently, the psychological consequences of obesity have seen an increased research effort as well (e.g. [2-4]).

Due to its high prevalence and thus overlap in affected individuals in the population with obesity, depression has been of particular interest regarding potential effects on psychological morbidity [5, 6]. Among adults, a meta-analysis of cross-sectional studies found an overall positive link between depression and obesity. However, subgroup analyses showed that the relationship between both conditions was more clearly present in women than in men [7]. Furthermore, a meta-analysis of longitudinal studies by Luppino and colleagues [8] has determined depression to be predictive of developing obesity. Additionally, they found that obesity increased the risk of depression and for clinically diagnosed depression. While a majority of studies suggests a positive association [9], findings on the relationship between obesity and depression have been inconsistent at times $[10,11]$. One suggested explanation for this circumstance is that the population with obesity is not homogenous. Previous research indicates that there are subgroups among the population with obesity which show increased psychopathology [12]. For example, individuals seeking weight loss treatment have been found to show elevated levels of psychiatric symptoms as well as depression (e.g. [13]).

Thus, several additional factors have been proposed to potentially determine which individuals with obesity will incur psychological distress. Next to the degree of obesity and social class, body image is considered as a potential mediator of the relationship between obesity and depression [12].

Body image refers to a person's perceptions and attitudes, including feelings, thoughts and behaviors, regarding their own body and appearance and is a multidimensional construct [14]. Appearance evaluation is one aspect of attitudinal body image and describes the subjective positive (or negative) evaluation of one's physical appearance [15]. Research has consistently found men to have a more positive body image than women [16]. Furthermore, studies on the relationship between obesity and body image found a more positive body image in normal-weight individuals compared to individuals with obesity (e.g. $[17,18])$ ). In addition, previous findings indicate that in the general population of individuals with obesity, positive body image tends to decrease as the degree of obesity rises [18]. However, there is no sufficient evidence of this association in men, and it does not seem to exist in subgroups like women seeking weight loss treatment [18]. In line with these findings, the association between negative body image and depressive symptoms appears to be independent from the degree of obesity [19]. Previous studies found negative body image to be related to increased depressive symptoms in women (e.g. [19,20]) and in men (e.g. [21]). Body image as a mediator has been explored in few studies so far, and Markowitzet al. [22] who reviewed the existing literature concluded that it might be an important mediator of depression in individuals with obesity.

In conclusion, identifying the underlying pathways linking obesity with depression and which individuals with obesity are at risk for developing depressive symptoms is particularly important for improving prevention and treatment efforts (e.g. [22]). The current study aims to contribute to existing knowledge on the topic by examining the role of the body image facet appearance evaluation in participants with obesity and explores the relationship of obesity, appearance evaluation, and depressive symptoms in a population sample of individuals with obesity. Moreover, differences in the association of obesity, appearance evaluation, and depressive symptoms between men and women with obesity were analyzed. Finally, appearance evaluation was investigated as a potential mediator of the relationship between obesity and depressive symptoms in men and women with obesity. 
Weinberger et al.: The Relationship between Weight Status and Depressive Symptoms

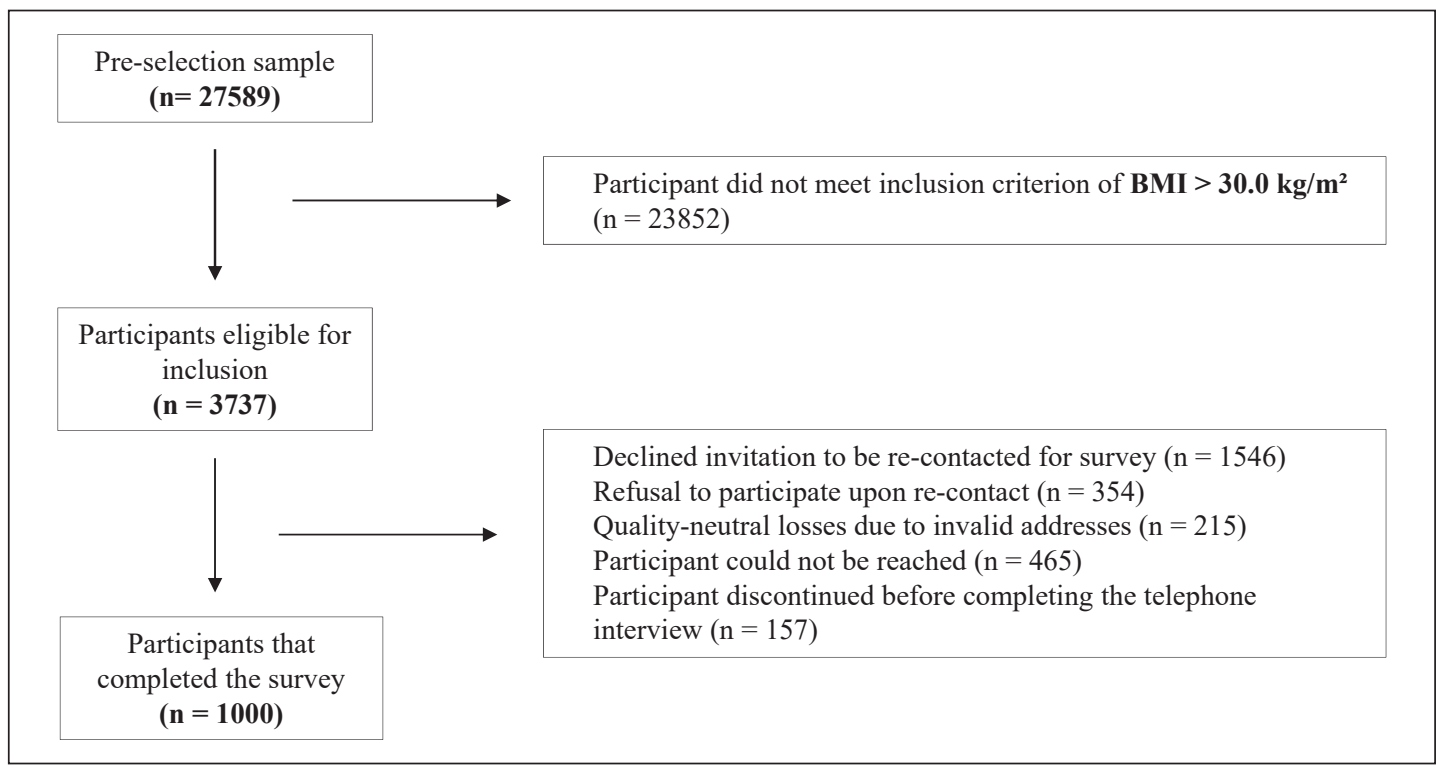

Fig. 1. Multi-level sampling process. The pre-selection sample consists of German-speaking individuals, aged 18 years and older.

\section{Material and Methods}

\section{Procedure}

This study was approved by the university's Ethics committee. Collection of data was conducted via a population-based telephone survey by a professional German market and opinion research institute in January 2015 to February 2015. Participants eligible for the study were invited to an interview that focused on "health, experience with an elevated body weight and general living environments of people in Germany". All participants gave verbal informed consent.

\section{Sampling Strategy}

A cross-sectional, multi-level sampling design was used. Sampling and telephone interviews were provided by the Forsa Institute for Social Research and Statistical Analysis (Berlin, Germany). Participants were preselected based on weight-specific questions as part of Forsa's continuous omnibus surveys, a daily telephone survey that yields empirical data with alternating focus on German-speaking individuals [23]. The German Market and Social Research Agency's (ADM) sampling system for telephone surveys was used, selecting potential interviewees by computer-generated random telephone numbers to ensure representativeness [24]. Trained research interviewers conducted the interviews, using a computer-assisted telephone interview (CATI) in German language. The pre-selection was followed by a main survey of individuals with obesity from the omnibus surveys who had agreed to be re-contacted and re-interviewed. The sampling process is illustrated in Figure 1.

\section{Participants}

Participants of this study were 451 women and 549 men with obesity. The majority of participants was categorized in obesity class I (63.8\% of women and $68.4 \%$ of men). Obesity class II was prevalent in approximately one-fourth of the sample (26.0\% of women and $24.1 \%$ of men) and obesity class III was found in $10.2 \%$ of women and $7.4 \%$ of men. Descriptive statistics of the sample are illustrated in Table 1.

\section{Instruments}

Participants were asked to provide basic sociodemographic information (e.g. age, gender, educational attainment) and to report their body height and weight at the beginning of the survey. To identify individuals seeking weight loss treatment, participants were asked to indicate whether they were currently undertaking 
Weinberger et al.: The Relationship between Weight Status and Depressive Symptoms in a Population Sample with Obesity: The Mediating Role of Appearance Evaluation

Table 1. Descriptive statistics (means and standard deviations) of BMI, appearance evaluation, depressive symptoms, age, education and efforts to reduce weight

\begin{tabular}{|c|c|c|c|c|}
\hline & $\begin{array}{l}\text { Total }(n=1,000) \\
\text { M (SD) }\end{array}$ & $\begin{array}{l}\text { Men }(n=549) \\
\text { M (SD) }\end{array}$ & $\begin{array}{l}\text { Women }(n=451) \\
\text { M (SD) }\end{array}$ & Difference \\
\hline BMI & $34.37(4.02)$ & $34.20(3.82)$ & $34.57(4.25)$ & $t(998)=-1.48$ \\
\hline Appearance evaluation ${ }^{\mathrm{a}}$ & $3.26(0.67)$ & $3.33(0.67)$ & $3.17(0.67)$ & $t(998)=3.86^{* * *}$ \\
\hline Depressive symptoms ${ }^{\mathrm{b}}$ & $5.39(4.24)$ & $4.84(3.95)$ & $6.06(4.47)$ & $t(998)=-4.57^{* * *}$ \\
\hline$\%$ of scores $>5$ & 35.2 & 33.3 & 37.5 & \\
\hline$\%$ of scores $>10$ & 14.6 & 11.5 & 18.5 & \\
\hline Age & $54.87(15.66)$ & $53.60(15.30)$ & $56.41(15.96)$ & $t(998)=-2.83^{* *}$ \\
\hline Education ${ }^{c}$ & $2.99(1.64)$ & $3.09(1.74)$ & $2.86(1.50)$ & $z=-1.21$ \\
\hline Weight reduction effort ${ }^{\mathrm{d}}$ & $1.60(0.49)$ & $1.59(0.49)$ & $1.62(0.49)$ & $\chi^{2}(1)=0.95$ \\
\hline$\%$ of "yes" & 60.2 & 58.9 & 61.9 & \\
\hline
\end{tabular}

${ }^{\mathrm{a}}$ Higher scores indicate greater satisfaction with physical appearance. ${ }^{\mathrm{b}} \mathrm{A}$ score between 5 and 10 indicates at least mild, a score of 10 or more, moderate levels of depressive symptoms. ${ }^{c}$ Four participants did not report educational information; range 1 = eighth grade or less to 7 = college degree. ${ }^{\mathrm{d}}$ One participant did not report information on current weight reduction efforts; $1=$ currently not undertaking efforts to reduce weight, $2=$ currently undertaking efforts to reduce weight. $* * p<0.01$. $* * * p<0.001$.

any efforts to reduce weight. Further, participants were interviewed about their experiences with an elevated body weight as well as their health (the Patient Health Questionnaire (PHQ-15; [26]), questions regarding weight management, appointments with general physicians/ medical specialist).

Multidimensional Body-Self Relations Questionnaire - Appearance Scales (MBSRQ - AS)

Body image was assessed using the German version of the Multidimensional Body-Self Relations Questionnaire - Appearance Scales (MBSRQ-AS; [25]). The MBSRQ-AS is a well-validated instrument [27] and consists of 34-items distributed among five subscales, which measure specific facets of appearance-related body image. The current study used the 7-item "Appearance Evaluation" subscale of the MBSRQ-AS, which is rated on a 5-point Likert scale ranging from "definitely disagree" to "definitely agree" and measures a subject's general feelings of satisfaction with their physical appearance. Scores on each item are averaged to create a mean score, with higher scores indicating greater satisfaction with appearance [25]. Overall internal consistency was acceptable $(\alpha=0.69)$. Additionally, analyses were run after the exclusion of two items. The shortened version of the subscale had a higher internal consistency.

The Patient Health Questionnaire - Depression Module (PHQ-9)

Depressive symptoms were assessed using the depression module of the Patient Health Questionnaire (PHQ-9), a self-report measure based on DSM-IV criteria for diagnosis of major depressive disorders [28]. Participants reported for each of the nine depressive symptoms if and how often the symptom had bothered them during the previous 2 weeks. The continuous variable is the sum of scores of the PHQ-9 items and ranges between 0 and 27. It is used to assess participants' self-reported severity of depressive symptoms, with scores of $5,10,15$ or $>15$ on the scale representing mild, moderate, moderately severe or severe levels of depressive symptoms, respectively. Previous studies found the PHQ-9 to be a reliable and valid measure of depression severity [28]. For the current study, the internal consistency was acceptable $(\alpha=0.76)$.

\section{Statistical Analyses}

Data was analyzed with SPSS 23.0.0 (IBM, Armonk, NY, USA) and weighted in regard to age, gender, and education of the population with obesity to control for bias. This variable was constructed by the research institute collecting the data. All analyses were run with and without the weight variable.

Pearson correlations were calculated to examine associations between the variables of interest (BMI, depressive symptoms, appearance evaluation), as well as between sociodemographic variables (age, gender; see Tables 2 and 3). Further, $t$ tests, Mann-Whitney $U$ tests and chi-square tests were used to analyze differences between men and women in all variables of interest (Table 1). 
Table 2. Correlations between BMI, appearance evaluation, depressive symptoms, and age in male participants with obesity

\begin{tabular}{|c|c|c|c|c|c|}
\hline & Variables & 1 & 2 & 3 & 4 \\
\hline 1 & BMI & - & $-0.240^{* * *}$ & $0.115^{* *}$ & $-0.184^{* * *}$ \\
\hline 2 & appearance evaluation $^{\mathrm{a}}$ & & - & $-0.305^{* * *}$ & 0.071 \\
\hline 3 & depressive symptoms & & & - & -0.013 \\
\hline 4 & age & & & & - \\
\hline
\end{tabular}

${ }^{a}$ Higher scores indicate greater satisfaction with physical appearance. ${ }^{* *} p<0.01 .{ }^{* * *} p<0.001$.

Table 3. Correlations between BMI, appearance evaluation, depressive symptoms, and age in female participants with obesity

\begin{tabular}{|c|c|c|c|c|c|}
\hline & Variables & 1 & 2 & 3 & 4 \\
\hline 1 & BMI & - & $-0.244^{* * *}$ & 0.048 & -0.066 \\
\hline 2 & appearance evaluation $^{\mathrm{a}}$ & & - & $-0.274^{* * *}$ & 0.087 \\
\hline 3 & depressive symptoms & & & - & -0.039 \\
\hline 4 & age & & & & - \\
\hline
\end{tabular}

${ }^{\text {a }}$ Higher scores indicate greater satisfaction with physical appearance. ${ }^{* * *} p<0.001$.

Appearance evaluation as a potential mediator was tested using the PROCESS method by Preacher and Hayes [29], drawing on 5,000 bootstrap samples from the dataset. Direct and indirect effects were estimated. Indirect effects were considered statistically significant at the 0.05 level of the $95 \%$ CI when the CI did not include zero. The mediation analysis was computed separately for women and men and adjusted for participants' age, education and whether they currently undertook any efforts to reduce their weight.

\section{Results}

Descriptive statistics of all variables of interest for the whole sample as well as for men and women separately are summarized in Table 1. Reporting of weighted data is not done, as no differences were observed. Also, since it did not yield different outcomes, results for appearance evaluation are reported for the complete scale.

Women reported significantly lower appearance satisfaction and significantly more depressive symptoms compared to men. On average, female participants were significantly older in comparison to the male participants.

In male and female participants, higher BMI was significantly related to lower appearance satisfaction. Moreover, a less positive body image was significantly associated with more depressive symptoms in both genders. A significant relationship between higher BMI and more depressive symptoms was only found in men. Tables 2 and 3 illustrate correlation coefficients between all variables of interest in male and female participants, respectively.

In the current sample, appearance evaluation mediated the association of BMI and depressive symptoms in men $(b=0.134$, $\mathrm{SE}=0.046, p=0.004)$. While no direct effect $(b=$ $0.064, \mathrm{SE}=0.045, p=0.160$ ) of BMI on depressive symptoms in men was observed, the bootstrapped unstandardized indirect effect was significant at 0.070 (SE $=0.018,95 \%$ CI $(0.040$, 0.110)). Male participants with higher BMIs reported lower appearance satisfaction and higher levels of depressive symptoms. 
Fig. 2. Mediation model BMI, appearance evaluation, and depressive symptoms in men with obesity. Path coefficients for the direct effect are included in parentheses and for the indirect effects outside parentheses. ${ }^{* *} p<$ 0.01 . $^{* * *} p<0.001$.

Weinberger et al.: The Relationship between Weight Status and Depressive Symptoms in a Population Sample with Obesity: The Mediating Role of Appearance Evaluation

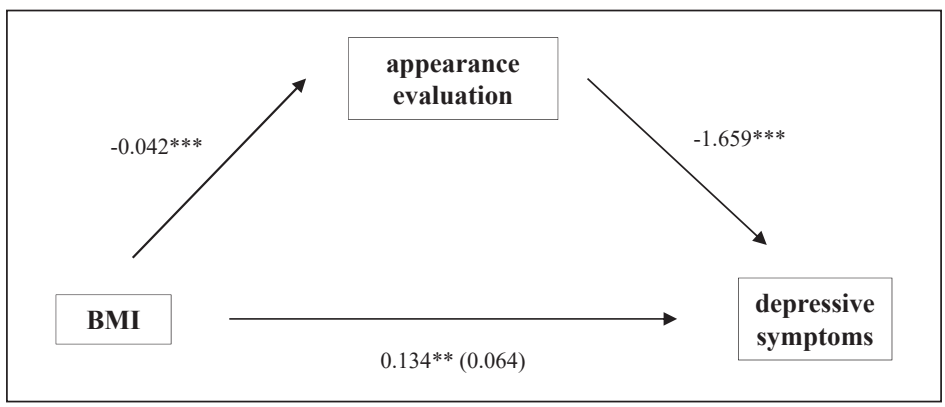

Because no significant relationship between BMI and depressive symptoms could be established in women, no mediation effect was determined. Figure 2 illustrates the mediating effect of appearance evaluation on the association of BMI and depressive symptoms in men.

Again, using weighted data did not yield different results.

\section{Discussion}

In the current study the relationship of BMI, appearance evaluation, and depressive symptoms was explored in a population sample of individuals with obesity. Differences in the association of all variables of interest between men and women with obesity were analyzed, and appearance evaluation was investigated as a potential mediator of the relationship between obesity and depressive symptoms.

Our analyses revealed male and female participants to be more satisfied with their physical appearance the lower their BMI was. Moreover, for both genders higher appearance satisfaction was related to fewer depressive symptoms. Men reported fewer depressive symptoms and a more positive appearance evaluation compared to women. Finally, while no mediation effect could be determined in women, appearance evaluation mediated the relationship between BMI and depressive symptoms in male participants.

The current findings regarding elevated weight status and appearance evaluation as well as appearance evaluation and depressive symptoms are in line with previous findings (e.g. $[18,19])$. In this context it is important to take into account Western society's tendency to emphasize thinness and beauty particularly in girls and women [30]. Media portrayal of the ideal physical appearance seems to have an influence on how individuals evaluate their own body [31]. This process of social comparison often results in a more negative body image in individuals with obesity since they tend to judge themselves as less attractive than the ideal (e.g. [32]). Considering how critical a factor appearance seems to be in how individuals - and especially girls and women - evaluate themselves, negative body image is theorized to directly contribute to depressive symptoms (e.g. [33]). In line with this assumption, particularly women with obesity report less satisfaction with their body and appearance compared to normal-weight women (e.g. [17]). Albeit research on the subject having mainly focused on women so far, a growing body of literature finds that men also report negative body image and that body image is a concern for them over the lifespan [34]. Moreover, the body image facet body dissatisfaction has been found to predict depressive mood in both girls and boys [35] and was related to depressive symptoms in both, men and women [36]. Differences between male and female participants in depressive symptoms and appearance evaluation also followed previously found patterns: studies consistently find higher prevalence of 
Weinberger et al.: The Relationship between Weight Status and Depressive Symptoms in a Population Sample with Obesity: The Mediating Role of Appearance Evaluation

depression (e.g. [37]) and a more negative view of body and appearance in women compared to men (e.g. [19]).

With regard to the missing link between obesity and depressive symptoms in women, our finding is surprising, but not unprecedented. In their meta-analysis, de Wit and colleagues [7] report that not all studies found an association between obesity and depression and that too few studies exist to draw conclusions on whether the severity of obesity influences depressive symptoms. One explanation as to why no significant relationship between BMI and depressive symptoms could be established in our female participants might be the reported severity of depressive symptoms. The majority of women in the current sample reported depressive symptom scores representing at least mild levels of depressive symptoms independently of their degree of obesity. This seems to provide further evidence that, rather than actual body size, psychological aspects like body image may be essential in the development of depressive symptoms in women with obesity. Stice and colleagues [33] for example reported similar findings in a sample of adolescent girls. Further, Gaskin and colleagues [38] found that among women controlling for body weight perception significantly weakened the relationship between measured weight and depression.

In contrast, the majority of men in our sample started to report more depressive symptoms only in the higher obesity classes. This observation is interesting in the context of previous findings on the relationship between obesity and depression in men. While - similarly to the current study - some studies do find a significant association (e.g. [39]), others only report a significant link of obesity and depression in women (e.g. [40]), and still others even suggest obesity to have a beneficial effect on mental health in men [41]. Thus, obesity class might be one of the keys to understanding this inconsistency, since research indicates particularly severe obesity to be associated with depression (e.g. [42]).

Further, a mediating effect of appearance evaluation could be determined in our male participants. This is in line with previous research, which found that body image mediated the relationship between obesity and depression (e.g. [36]). Moreover, the current findings underline the important role of body image facets in the obesity-depression association and have implications regarding treatment efforts. There is a certain amount of interaction in the treatment of obesity and in that of depression, with improvements in one condition having a positive effect on the other [6]. However, the mutual interference is not always beneficial. For instance, there are indications that dieting might deteriorate mood, and weight gain is one common side effect of several psychotropic medications used in the treatment of depression [22]. As such, efforts to reduce adverse effects of either obesity or depression need to take the reciprocal relationship between the two conditions into account. Furthermore, rather than health, negative body image is often the most motivating factor for individuals with obesity to seek treatment [19]. As such, interventions addressing body image might also improve psychological distress and eating behaviors, which in turn could benefit obesity management in general [36]. In line with this assumption, there is evidence of a reciprocal effect between improvements in body image and weight reduction during obesity treatment [43].

Interpretation of our findings should take the following limitations into consideration. First, the cross-sectional design of the current study calls for particular care interpreting the links between the analyzed variables as causal in nature. While the current results are similar to previous studies, whether the relationship between depression and appearance evaluation is reciprocal or in which direction influence of one variable on the other is more significant, ultimately needs to be determined using longitudinal data.

Moreover, self-reported height and weight data was used to calculate BMI. While participants tend to underestimate their weight and overestimate their height [44], previous research suggests a high correlation between self-reported and directly measured anthropometric data [45]. Additionally, previous research of the association between obesity and 
Weinberger et al.: The Relationship between Weight Status and Depressive Symptoms in a Population Sample with Obesity: The Mediating Role of Appearance Evaluation

depression has included both self-report and actual assessment of BMI, with no indication of influence of the measuring method $[9,46]$.

Further, due to time and budget constraints with regard to the telephone survey, assessment of body image was constrained to one construct (appearance evaluation) rather than to its full, multidimensional nature. As such, the study assessed e.g. participants' estimation of their attractiveness, the way our participants with obesity feel in their clothing, or how satisfied they are with their physical build. Thus, the current results provide information regarding the subjective evaluation of appearance rather than body image in general. Consequently, complete assessment of body image in participants with obesity (including satisfaction, perception, etc.) could be a valuable endeavor for future studies on the subject.

In addition, questions pertaining to psychotropic medication use and its potential influence on depressive symptoms as well as weight status could not be assessed. However, evidence indicates that accounting for psychotropic drug use might not affect the link between obesity and depression [9].

Finally, depressive symptoms were assessed by self-report measure rather than a full clinical assessment. Previous research indicates that misclassification of depression might diminish the association between obesity and depression [47]. However, compared to standard clinician assessment, the validity of the PHQ was found to be equal if not better than alternative measures used in past studies [28, 47].

\section{Conclusion}

The current study contributes to existing research on the topic in two important ways: Firstly, our findings provide further evidence for the importance of appearance satisfaction in the relationship between obesity and depressive symptoms in adult individuals with obesity. Secondly, our study adds to the still growing literature on the nature of body image in men with obesity. Additional studies are needed to ascertain which role other dimensions of body image, e.g. body area satisfaction or body size perception, play in the relationship between BMI and depressive symptoms in individuals with obesity. Future research should also include longitudinal studies on the topic and ideally examine diverse populations with obesity.

\section{Funding}

This work was supported by the Federal Ministry of Education and Research (BMBF), Germany, FKZ: 01E01501. We acknowledge support from the German Research Foundation (DFG) and University of Leipzig within the program of Open Access Publishing.

\section{Disclosure Statement}

All authors declare no conflict of interest. 
Weinberger et al.: The Relationship between Weight Status and Depressive Symptoms in a Population Sample with Obesity: The Mediating Role of Appearance Evaluation

\section{References}

1 Haslam DW, James WP. Obesity. Lancet. 2005 Oct;366(9492):1197-209.

2 Carpenter KM, Hasin DS, Allison DB, Faith MS. Relationships between obesity and DSM-IV major depressive disorder, suicide ideation, and suicide attempts: results from a general population study. Am J Public Health. $2000 \mathrm{Feb}$;0(2):251-7.

3 Simon GE, Von Korff M, Saunders K, Miglioretti DL, Crane PK, van Belle G, et al. Association between obesity and psychiatric disorders in the US adult population. Arch Gen Psychiatry. 2006 Jul;63(7):824-30.

4 Sarwer DB, Polonsky HM. The Psychosocial Burden of Obesity. Endocrinol Metab Clin North Am. 2016 Sep; 45(3):677-88.

5 Atlantis E, Baker M. Obesity effects on depression: systematic review of epidemiological studies. Int J Obes. 2008 Jun;32(6):881-91.

6 Stunkard AJ, Faith MS, Allison KC. Depression and obesity. Biol Psychiatry. 2003 Aug;54(3):330-7.

7 de Wit L, Luppino F, van Straten A, Penninx B, Zitman F, Cuijpers P. Depression and obesity: a meta-analysis of community-based studies. Psychiatry Res. 2010 Jul;178(2):230-5.

8 Luppino FS, de Wit LM, Bouvy PF, Stijnen T, Cuijpers P, Penninx BW, et al. Overweight, obesity, and depression: a systematic review and meta-analysis of longitudinal studies. Arch Gen Psychiatry. 2010 Mar;67(3):220-9.

9 Gavin AR, Simon GE, Ludman EJ. The association between obesity, depression, and educational attainment in women: the mediating role of body image dissatisfaction. J Psychosom Res. 2010 Dec;69(6):573-81.

10 John U, Meyer C, Rumpf HJ, Hapke U. Relationships of psychiatric disorders with overweight and obesity in an adult general population. Obes Res. 2005 Jan;13(1):101-9.

11 Jorm AF, Korten AE, Christensen H, Jacomb PA, Rodgers B, Parslow RA. Association of obesity with anxiety, depression and emotional well-being: a community survey. Aust N Z J Public Health. 2003;27(4):434-40.

12 Friedman MA, Brownell KD. Psychological correlates of obesity: moving to the next research generation. Psychol Bull. 1995 Jan;117(1):3-20.

13 Goldsmith SJ, Anger-Friedfeld K, Rudolph D, Boeck M, Aronne L. Psychiatric illness in patients presenting for obesity treatment. Int J Eat Disord. 1992;12(1):63-71.

14 Cash TF. Body image: Cognitive behavioral perspectives on body image. In: Cash TF, Pruzinsky T, editors. Body Images: A Handbook of Theory, Research, and Clinical Practice. NY: Guilford Press; 2002. pp. 38-46.

15 Cash TF. Body-image attitudes: evaluation, investment, and affect. Percept Mot Skills. 1994 Jun;78(3 Pt 2): 1168-70.

16 Cash TF, Roy RE: Pounds of flesh: Weight, gender, and body images. In: Sobal J, Maurer D, editors. Interpreting weight: The social management of fatness and thinness. New York: Aldine de Gruyter; 1999;209-228.

17 Weinberger NA, Kersting A, Riedel-Heller SG, Luck-Sikorski C. Body Dissatisfaction in Individuals with Obesity Compared to Normal-Weight Individuals: A Systematic Review and Meta-Analysis. Obes Facts. 2016;9(6): 424-41.

18 Schwartz MB, Brownell KD. Obesity and body image. Body Image. 2004 Jan;1(1):43-56.

19 Sarwer DB, Thompson JK, Cash TF. Body image and obesity in adulthood. Psychiatr Clin North Am. 2005 Mar; 28(1):69-87.

20 Sarwer DB, Wadden TA, Foster GD. Assessment of body image dissatisfaction in obese women: specificity, severity, and clinical significance. J Consult Clin Psychol. 1998 Aug;66(4):651-4.

21 Olivardia R, Pope HG Jr, Borowiecki JJ 3rd, Cohane GH. Biceps and Body Image: The Relationship Between Muscularity and Self-Esteem, Depression, and Eating Disorder Symptoms. Psychol Men Masc. 2004;5(2):11220.

22 Markowitz S, Friedman MA, Arent SM. Understanding the Relation Between Obesity and Depression: Causal Mechanisms and Implications for Treatment. Clin Psychol Sci Pract. 2008;15(1):1-20.

23 forsa Gesellschaft für Sozialforschung und Statistische Analysen mbH. Methoden. https://www.forsa.de/ methoden/.

24 ADM Arbeitskreis Deutscher Markt- und Sozialforschungsinstitute e. V.: Die ADM Stichproben für Telefonbefragungen. https://www.adm-ev.de/index.php?id =77\&L $=0$.

25 Cash TF. The multidimensional body-self relations questionnaire (3rd ed.): (Available from the author at www.body-images.com), 2000.

26 Kroenke K, Spitzer RL, Williams JB. The PHQ-15: validity of a new measure for evaluating the severity of somatic symptoms. Psychosom Med. 2002 Mar-Apr;64(2):258-66. Available from: https://journals.lww. com/psychosomaticmedicine/Fulltext/2002/03000/The_PHQ_15_Validity_of_a_New_Measure_for.8.aspx

27 Vossbeck-Elsebusch AN, Waldorf M, Legenbauer T, Bauer A, Cordes M, Vocks S. German version of the Multidimensional Body-Self Relations Questionnaire - Appearance Scales (MBSRQ-AS): confirmatory factor analysis and validation. Body Image. 2014 Jun;11(3):191-200.

28 Kroenke K, Spitzer RL, Williams JB. The PHQ-9: validity of a brief depression severity measure. J Gen Intern Med. 2001 Sep;16(9):606-13.

29 Preacher KJ, Hayes AF. Asymptotic and resampling strategies for assessing and comparing indirect effects in multiple mediator models. Behav Res Methods. 2008 Aug;40(3):879-91.

30 Murnen SK, Don BP. Body Image and Gender Roles A2 - Cash, Thomas; in T1-Body Image and Gender Roles A2-Cash T (ed): Encyclopedia of Body Image and Human Appearance. Oxford, Academic Press, 2012, pp 128-134. 
Weinberger et al.: The Relationship between Weight Status and Depressive Symptoms in a Population Sample with Obesity: The Mediating Role of Appearance Evaluation

31 Groesz LM, Levine MP, Murnen SK. The effect of experimental presentation of thin media images on body satisfaction: a meta-analytic review. Int J Eat Disord. 2002 Jan;31(1):1-16.

32 Tiggemann M, Slater A. Thin ideals in music television: a source of social comparison and body dissatisfaction. Int J Eat Disord. 2004 Jan;35(1):48-58.

33 Stice E, Hayward C, Cameron RP, Killen JD, Taylor CB. Body-image and eating disturbances predict onset of depression among female adolescents: a longitudinal study. J Abnorm Psychol. 2000 Aug;109(3):438-44.

34 McCabe MP, Ricciardelli LA. Body image dissatisfaction among males across the lifespan: a review of past literature. J Psychosom Res. 2004 Jun;56(6):675-85.

35 Paxton SJ, Neumark-Sztainer D, Hannan PJ, Eisenberg ME. Body dissatisfaction prospectively predicts depressive mood and low self-esteem in adolescent girls and boys. J Clin Child Adolesc Psychol. 2006 Dec; 35(4):539-49.

36 Friedman KE, Reichmann SK, Costanzo PR, Musante GJ. Body image partially mediates the relationship between obesity and psychological distress. Obes Res. 2002 Jan;10(1):33-41.

37 Piccinelli M, Wilkinson G. Gender differences in depression. Critical review. Br J Psychiatry. 2000 Dec;177(06): 486-92.

38 Gaskin JL, Pulver AJ, Branch K, Kabore A, James T, Zhang J. Perception or reality of body weight: which matters to the depressive symptoms. J Affect Disord. 2013 Sep;150(2):350-5.

39 Herva A, Laitinen J, Miettunen J, Veijola J, Karvonen JT, Läksy K, et al. Obesity and depression: results from the longitudinal Northern Finland 1966 Birth Cohort Study. Int J Obes. 2006 Mar;30(3):520-7.

40 Scott KM, McGee MA, Wells JE, Oakley Browne MA. Obesity and mental disorders in the adult general population. J Psychosom Res. 2008 Jan;64(1):97-105.

41 Magallares A, Pais-Ribeiro JL. Mental health and obesity: a meta-analysis. Appl Res Qual Life. 2014;9(2):295308.

42 Onyike CU, Crum RM, Lee HB, Lyketsos CG, Eaton WW. Is obesity associated with major depression? Results from the Third National Health and Nutrition Examination Survey. Am J Epidemiol. 2003 Dec;158(12):113947.

43 Palmeira AL, Markland DA, Silva MN, Branco TL, Martins SC, Minderico CS, et al. Reciprocal effects among changes in weight, body image, and other psychological factors during behavioral obesity treatment: a mediation analysis. Int J Behav Nutr Phys Act. 2009 Feb;6(1):9.

44 Connor Gorber S, Tremblay M, Moher D, Gorber B. A comparison of direct vs. self-report measures for assessing height, weight and body mass index: a systematic review. Obes Rev. 2007 Jul;8(4):307-26.

45 Niedhammer I, Bugel I, Bonenfant S, Goldberg M, Leclerc A. Validity of self-reported weight and height in the French GAZEL cohort. Int J Obes Relat Metab Disord. 2000 Sep;24(9):1111-8.

46 Jeffery RW, Finch EA, Linde JA, Simon GE, Ludman EJ, Operskalski BH, et al. Does clinical depression affect the accuracy of self-reported height and weight in obese women? Obesity (Silver Spring). 2008 Feb;16(2):473-5.

47 Simon GE, Ludman EJ, Linde JA, Operskalski BH, Ichikawa L, Rohde P, et al. Association between obesity and depression in middle-aged women. Gen Hosp Psychiatry. 2008 Jan-Feb;30(1):32-9. 